\title{
Statistical Effects from Hipparcos Astrometry
}

\author{
Frédéric Arenou \\ DASGAL/URA 335 du C.N.R.S. - Observatoire de Paris-Meudon, \\ F-92195 Meudon Cedex, France - Frederic.Arenou@obspm.fr
}

\author{
Xavier Luri \\ Departament d'Astronomia i Meteorologia, Universitat de Barcelona, \\ Avda. Diagonal 647, E-08028, Barcelona, Spain-xluri@am.ub.es
}

\begin{abstract}
The Hipparcos astrometry is used mainly for the derivation of stellar physical quantities such as luminosity, masses and velocity. However, sample selections on data with observational errors or an intrinsic dispersion may lead to biased estimates, especially when the error distributions are non-Gaussian. We review the classical biases and the ways to avoid them through the use of statistical methods.
\end{abstract}

\section{Introduction}

The primary way to calibrate absolute magnitudes is through the use of Hipparcos (ESA 1997) parallaxes. Except the rare stars with a very small relative parallax error, it is actually hard to avoid statistical biases in luminosity calibrations (see e.g. Arenou \& Luri 1999 for a detailed discussion). Many other biases can occur, due to an incorrect extinction model, duplicity, etc. We will focus here on the absolute magnitudes and we refer to Arenou \& Palasi (2000) for what concerns the masses derived from the Hipparcos astrometry.

\section{Non-linear Functions}

The first problem is related to the fact that the parameters of interest (distance, absolute magnitudes, velocity) are usually non-linear functions of the parallax. Due to this, on average the "observed" (derived from the parallaxes) absolute magnitudes of a sample will be biased, that is to say, its distribution will not represent the true distribution, leading for instance to a wrong estimation of the mean absolute magnitude. Moreover, the error bar can be highly asymmetric and of about two magnitudes for a $100 \%$ relative error. And, of course, negative parallaxes can not be used directly.

The only way to avoid this bias is to work in a plane of linear functions of parallaxes, whose error distribution is nearly Gaussian. For example, instead of using a classical HR diagram, it is much more sensible, from a statistical point of view, to use an "astrometric" HR diagram with colour as abscissa and the "reduced parallax" $a_{V}=10^{0.2 M_{V}}=\pi 10^{\frac{m_{V}+5}{5}}$ on the ordinate. In such a 
diagram, even stars with negative parallax can be plotted, and the error bars are symmetric. Except for possible Malmquist (1936) effects (Sect. 5) no other bias is expected.

\section{Correlation and Truncation}

The second source of bias arises from correlations in the observational data. When random errors in two variables are correlated, the correlation can produce effects that in some cases may be confused with a systematic error. For instance, plotting $\pi_{\text {Hip }}-\pi_{\text {photo }}$ versus $\pi_{\text {photo }}$ shows a trend which could be attributed to some systematic error in the photometric calibration; however this effect is easy to reproduce using a Gaussian error law for the absolute magnitude and some spatial model for the parallax distribution. A more extreme example can be shown by plotting $M_{\text {Hip }}-M_{\text {photo }}$ versus $M_{\text {Hip }}$; this time the effect will depend on the asymmetrical error bars on $M_{\mathrm{Hip}}$, on the luminosity distribution and with a probable Malmquist (1936) effect, so that nothing useful can be deduced from such graphs. The only way around is to plot uncorrelated variables.

It is important to notice that a correlation does not introduce a bias by itself. A bias occurs on the average of the ordinate variable when a truncation is done on the abscissa variable. The classical example is the so-called Lutz-Kelker bias (Lutz \& Kelker 1973), when $M_{\text {Hip }}$ is plotted against $\frac{\sigma_{\pi}}{\pi_{\text {Hip }}}$.

\section{The Lutz-Kelker Bias}

The problem arises when stars are selected by imposing a (usually small) limit to the relative parallax error, in the hope of getting the most precise absolute magnitudes. Since this is a truncation on the observed parallax, whose distribution is not uniform, the resulting mean absolute magnitude is biased. This mean absolute magnitude (or, correspondingly, the mean parallax) should then be corrected by an amount which depends on the sample distribution, e.g. $\Delta M=0.43$ mag at $\frac{\sigma_{\pi}}{\pi_{\text {Hip }}}=0.175$ for a distance-limited sample. A common misunderstanding of the problem is to think that this is an individual (per star) correction, improving or correcting in some way the individual parallaxes. However, the individual parallaxes are (unless some observational problem is present, which is not the case for Hipparcos) unbiased, and the correction is applied to compensate for the (biasing) selection process. The Lutz-Kelker correction (Lutz \& Kelker 1973) may have a very large confidence interval (Koen 1992), and it also depends on the assumed spatial distribution. This means that the corrected mean absolute magnitude may be both biased (if the adopted model is incorrect) and imprecise. One should come back to the initial cause of the bias, which is a truncation based on the observed parallax and avoid the truncation. The bias would be avoided and all the available data will be used.

\section{The Malmquist Bias}

Up to now, we have dealt with biases created by the user of the data, and we pointed how to avoid them. However, the way the initial sample was selected 
has also to be taken into account. In most Catalogues, and in particular in Hipparcos, the initial sample is magnitude-limited, whereas usually one would like a distance-limited sample for magnitude calibrations, because due to the intrinsic dispersion of the absolute magnitude combined with the truncation in apparent magnitude, intrinsically brighter stars are over-represented in this kind of sample. This introduces a bias not only in the mean absolute magnitude, but more generally in all the moments of the distribution, and in particular the dispersion, which would be needed to correct the bias. This means that the residuals of an absolute magnitude calibration, after subtraction of the dispersion due to the parallax errors, will give a biased value of the intrinsic dispersion. There can be a sort of unbiased plateau, a distance up to which the sample will be complete, and from which unbiased statistics can be computed, but this will reduce the amount of useful data. In order to correct the bias, the true spatial distribution must be known. In the case of an exponential distribution perpendicular to the galactic plane, the bias will depend on the limiting magnitude, the scale height and the magnitude dispersion, and will thus be different from the classical $1.38 \sigma^{2}$ correction (Luri et al. 1993). The distribution of the absolute magnitude around the mean value is also classically considered Gaussian in the estimations of the bias, whereas the effects of evolution, metallicity and rotation lead to an asymmetric distribution.

The correction of this bias is thus not obvious. In the best case, adding enough parameters to a calibration allows one to get a small dispersion in absolute magnitude, and thus a small bias. Otherwise, one has to rely on a parametrical model.

\section{Calibration Methods}

If a calibration is done using parametrical methods, a maximum likelihood analysis allows us to make use of all the available information, including the sample selection function. Such an analysis was done first by Jung (1971) and the method has been further improved by Ratnatunga \& Casertano (1991) and Luri et al. (1996). We will not discuss the methods which are intended to correct Lutz-Kelker bias, since we have shown that this bias may be avoided. In the parametric methods, the probability that a star has been observed with its parallax, proper motion, magnitude, etc, is computed with the help of a kinematical model, a luminosity error distribution and a spatial distribution. If these models are correct the luminosity calibration will be optimal and unbiased, since each possible truncation of the above cited distributions may be taken explicitly into account. On the contrary, biased results will be obtained if the sample does not follow the assumed distribution.

Among non-parametrical methods, the first one proposed simply works in the parallax domain and gives asymptotically unbiased estimates since the conversion to absolute magnitudes is done on the mean reduced parallax. This method has been used by Roman (1952), justified by Jung (1971) and further improved by van Leeuwen \& Evans (1998) using the intermediate Hipparcos data. Other non-parametrical methods, using a transformation of the observed parallax, have been suggested recently. For instance Smith \& Eichhorn (1996) use a function of the observed parallax for computing the mean distance or 
mean absolute magnitude. Moreover Smith (2000) improved the function and showed that it could be more precise than the reduced parallax method. The performance of the reduced parallax method will be improved when the intrinsic magnitude dispersion is taken into account in the weights used to compute the mean value. Also if the absolute magnitude errors are assumed Gaussian, a correction $-0.23 \sigma_{M}^{2}$ to the mean magnitude should be applied, due to the asymmetry of the transformation (Jung 1971); for the reason noted above, however, the reduced parallax should have an error distribution more symmetric than the absolute magnitude, and this correction would not be applied.

\section{Sample Selection}

To elaborate a luminosity calibration, one would like to use a sample representative of the parent population, that is, complete in a statistical sense, or a sample for which one knows exactly its selection function. When using Hipparcos stars, the only sample for which we know the exact selection function is the Survey, which is magnitude-limited. For fainter stars the exact selection will be hard (if possible at all) to know. To be conservative, a luminosity calibration should then use only the Survey. Extracting a volume-limited sample from the Survey in order to avoid the Malmquist bias is not easy. For instance, selecting a sample with $\frac{\sigma_{\pi}}{\pi_{\text {Hip }}}$ smaller than some value does not give a distance-limited sample since $\sigma_{\pi}$ may vary by a factor 3 with the magnitude. Or using a photometric parallax to select a sample, since $\pi_{\text {photo }}=10^{-0.2(m-M)-1}>\pi_{\min }$ is equivalent to $m<M-5 \log \pi_{\min }-5$, creating once again a magnitude-limited sample.

\section{References}

Arenou, F., \& Luri X. 1999, in ASP Conf. Ser. Vol. 167, Harmonizing Cosmic Distance Scales in a Post-Hipparcos Era, ed. D. Egret \& A. Heck (San Francisco: ASP), 13

Arenou, F., \& Palasi J. 2000, in IAU Symp. 202, Planetary Systems in the Universe, ed. A. Penny et al., in press

ESA 1997, The Hipparcos Catalogue, ESA SP-1200, vol. I, Sect. 2.3

Jung, J. 1971, A\&A, 11, 351

Koen, C. 1992, MNRAS, 256, 65

van Leeuwen, F., \& Evans, D. 1998, A\&AS, 130, 157

Luri, X., Mennessier, M.O., Torra, J. \& Figueras, F. 1993, A\&AS, 267, 305L

Luri, X., Mennessier, M.O., Torra, J., \& Figueras, F. 1996, A\&AS, 117, 405

Lutz, T.E., \& Kelker, D.H. 1973, PASP, 85, 573

Malmquist, K.G. 1936, Meddel. Stockholm Obs., 26

Ratnatunga, K.U., \& Casertano, S. 1991, AJ, 101, 1075

Roman, N. 1952, ApJ, 116, 122

Smith, H., \& Eichhorn, H. 1996, MNRAS, 281, 211

Smith, H. 2000, in Proceedings of the Fifth Alexander von Humboldt Colloquium on Celestial Mechanics, ed. R. Dvorak \& J. Henrard, in press 